\title{
UNIFORM SETS FOR INFINITE MEASURE-PRESERVING SYSTEMS
}

\author{
HISATOSHI YUASA
}

\begin{abstract}
The concept of a uniform set is introduced for an ergodic, measurepreserving transformation on a non-atomic, infinite Lebesgue space. The uniform sets exist as much as they generate the underlying $\sigma$-algebra. This leads to the result that any ergodic, measure-preserving transformation on a non-atomic, infinite Lebesgue space is isomorphic to a minimal homeomorphism on a locally compact metric space which admits a unique, up to scaling, invariant Radon measure.
\end{abstract}

\section{INTRODUCTION}

The present work concerns constructing a topological model of a given ergodic measure-preserving system. R. I. Jewett [12] showed that any weakly mixing measure-preserving transformation on a non-atomic, Lebesgue probability space is (measure-theoretically) isomorphic to a strictly ergodic homeomorphism on a Cantor set, which was extended by W. Krieger [14] to all ergodic systems. This model theorem is the so-called Jewett-Krieger Theorem. G. Hansel and J. P. Raoult [10] emphasized that uniform partitions play important roles in proving JewettKrieger Theorem. B. Weiss [18, 19] added a categorical taste to the model theorem: if $\pi: \mathbf{Y}_{1} \rightarrow \mathbf{Y}_{2}$ is a factor map between ergodic systems and if $\mathbf{X}_{2}$ is a strictly ergodic model of $\mathbf{Y}_{2}$, then there exists a strictly ergodic model $\mathbf{X}_{1}$ of $\mathbf{Y}_{1}$ such that the diagram

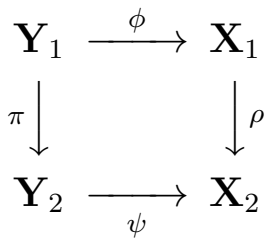

commutes, where $\phi$ and $\psi$ are isomorphisms and $\rho$ is a topological factor map. In connection with topological orbit equivalence [7], N. Ormes [17] showed a generalization of Jewett-Krieger Theorem that any ergodic system has a topological model which is orbit equivalent to a given Cantor minimal system with a given invariant probability measure. Along this line, H. Matui [15] achieved a model theorem which realizes an ergodic system as a minimal homeomorphisms on a locally compact metric space. I. Kornfeld and N. Ormes [13] generalized Ormes' model theorem for families of ergodic systems. Strictly ergodic models for ergodic actions by groups other than $\mathbb{Z}$ were obtained for $\mathbb{R}$-action by K. Jacobs [11], M. Denker and E. Eberlein [5], and for free actions by the commutative groups by B. Weiss [18].

2000 Mathematics Subject Classification. Primary 37A05, 37B05; Secondary .

Key words and phrases. infinite measure-preserving system, ergodicity, Kakutani-Rohlin tower, topological model, almost minimality, locally compact Cantor minimal system, invariant Radon measure. 
All of the above-mentioned model theorems target the ergodic transformations preserving probability measures. It is the present work that initiates a model theorem of ergodic, infinite measure-preserving systems. It is actually proved in Theorem 4.5 that an ergodic, infinite measure-preserving system has a topological model of a minimal homeomorphism on a locally compact metric space admitting a unique, up to scaling, invariant Radon measure. Since our strategy for proving the theorem is to follow the line of B. Weiss [18, 19] (see also [8]), the concept of a uniform set should be formalised suitably for the infinite measure-preserving case. This is accomplished by Definition 4.1. Lemmas 4.3 and 4.4 are crucial to prove Theorem 4.5. It follows from the lemmas that the ergodic system can be approximated by a refining sequence of uniform partitions which generate the underlying $\sigma$-algebra. A uniform partition is a finite partition with a unique atom of infinite measure whose atoms of finite measure are all uniform. The uniform partition has an advantage to give rise to an almost minimal factor admitting a unique, up to scaling, invariant Radon measure. This fact is verified in virtue of Proposition 3.2. The proposition characterizes a homeomorphism on a locally compact metric space admitting a unique, up to scaling, Radon measure. As an immediate consequence of Theorem 4.5, it holds that an ergodic, infinite measure-preserving system is isomorphic to the Vershik map arising from an almost simple, ordered Bratteli diagram in the sense of [4]. Unfortunately, a categorical realization of a factor map between infinite measure-preserving systems has not been achieved yet. This problem is the infinite measure counterpart of the categorical model theorem of B. Weiss [18, 19].

The author thanks Professor T. Hamachi for letting him know the existence of an extension [16] of Kolmogorov consistency theorem to infinite measure spaces.

\section{RATIO ERGODIC THEOREM ON TOWERS}

In this section, we briefly review some basic concepts and facts concerning transformations on measure spaces which preserve measures; in particular, finite partitions, symbolic factors associated with them and Kakutani-Rohlin partitions. Without explicitly stating, we assume any relations among measurable subsets of a measure space, or any properties of maps between measure spaces and so on are taken to hold up to sets of measure zero.

If a measure space $(Y, \mathcal{C}, \nu)$ is isomorphic to the measure space of real numbers equipped with the $\sigma$-algebra of Lebesgue measurable subsets and Lebesgue measure, then we call $(Y, \mathcal{C}, \nu)$ a non-atomic, infinite Lebesgue space. If $X$ is a complete separable metric space and $\mu$ is a non-atomic, infinite, $\sigma$-finite measure on the Borel $\sigma$-algebra $\mathcal{B}$ of $X$, then $\left(X, \mathcal{B}_{\mu}, \mu\right)$ is isomorphic to a non-atomic, infinite Lebesgue space, where $\mathcal{B}_{\mu}$ is the completion of $\mathcal{B}$ under $\mu$. See for details [1, Chapter 1].

Let $(Y, \mathcal{C}, \nu)$ be a non-atomic, infinite Lebesgue space. A bijection $T: Y \rightarrow$ $Y$ is said to be bi-measurable if both of $T$ and $T^{-1}$ are measurable. Suppose a bi-measurable bijection $T: Y \rightarrow Y$ preserves the measure $\nu$, i.e. $\nu \circ T^{-1}(E):=$ $\nu\left(T^{-1} E\right)=\nu(E)$ for all $E \in \mathcal{C}$. The measure $\nu$ is also said to be $T$-invariant. We then call $(Y, \mathcal{C}, \nu, T)$ an infinite measure-preserving system. If $\nu(B)=0$ or $\nu(Y \backslash$ $B)=0$ for any $T$-invariant set $B \in \mathcal{C}$, then $T$ is said to be ergodic. The ergodicity implies that $T$ is aperiodic, i.e. the orbit $\operatorname{Orb}_{T}(y):=\left\{T^{n} y \mid n \in \mathbb{Z}\right\}$ of any point $y \in$ $Y$ is infinite. We refer to a set $\left\{T^{i} y \mid m \leq i \leq n\right\}$ with $m \leq n$ as a section of $\operatorname{Orb}_{T}(y)$. Symbolic examples over finite states of ergodic, infinite measure-preserving systems 
are recently obtained by [2, 3, 9, 21]. Throughout the remainder of this paper, we assume $(Y, \mathcal{C}, \nu, T)$ is an ergodic, infinite measure-preserving system. Suppose $\left(Y^{\prime}, \mathcal{C}^{\prime}, \nu^{\prime}, T^{\prime}\right)$ is an infinite measure-preserving system. If there exists a measurable surjection $\phi: Y \rightarrow Y^{\prime}$ such that $\nu^{\prime}=\nu \circ \phi^{-1}$ and $\phi \circ T=T^{\prime} \circ \phi$, then $\phi$ and $T^{\prime}$ are called a factor map and a factor of $T$, respectively. Then, $T^{\prime}$ is necessarily ergodic. If in addition $\phi$ is injective, then $\left(Y^{\prime}, \mathcal{C}^{\prime}, \nu^{\prime}, T^{\prime}\right)$ is said to be isomorphic to $(Y, \mathcal{C}, \nu, T)$

An element of a partition of $Y$ into measurable subsets is called an atom. A partition $\beta$ is said to refine a partition $\alpha$ if each atom of $\alpha$ is a union of atoms of $\beta$. If $\alpha$ is written as $\left\{A_{1}, A_{2}, \ldots, A_{N}\right\}$ with $N \geq 2$ and if $\alpha$ has a unique atom of infinite measure, then we call $\alpha$ a finite partition. We always assume the unique atom has index 1, i.e. $\nu\left(A_{1}\right)=\infty$. Let $K_{\alpha}$ denote the set $Y \backslash A_{1}$. For another finite partition $\beta$, we define the join $\alpha \vee \beta$ to be a finite partition $\{A \cap B \mid A \in \alpha, B \in \beta\}$. This definition and notation may be extended naturally to the join of a finite number of finite partitions. A finite partition $\bigvee_{i=m}^{n} T^{-i} \alpha$ with $m \leq n$ is denoted by $\alpha_{m}^{n}$. If $\beta$ is written as $\left\{B_{1}, B_{2}, \ldots, B_{N}\right\}$, then we set $d(\alpha, \beta)=\sum_{i \neq 1} \nu\left(A_{i} \triangle B_{i}\right)$. If a sequence $\left\{\alpha_{n}\right\}_{n \in \mathbb{N}}$ of finite partitions is a Cauchy sequence in $d$, where $\sharp \alpha_{n}$ is assumed constant, then there exists a finite partition $\alpha_{0}$ such that $\lim _{n \rightarrow \infty} d\left(\alpha_{n}, \alpha_{0}\right)=0$.

Regard the index set $\mathfrak{A}_{\alpha}=\{1,2, \ldots, N\}$ of the finite partition $\alpha$ as a finite alphabet. Define a measurable map $\phi_{\alpha}: Y \rightarrow \mathfrak{A}_{\alpha}^{\mathbb{Z}}$ so that $T^{i} y \in A_{\phi_{\alpha}(y)_{i}}$ for every $i \in \mathbb{Z}$. An infinite, $\sigma$-finite, Borel measure $\hat{\mu}_{\alpha}:=\nu \circ \phi_{\alpha}{ }^{-1}$ is invariant under the left shift on $\mathfrak{A}_{\alpha}^{\mathbb{Z}}$. The support $\hat{X}_{\alpha}$ of $\hat{\mu}_{\alpha}$, i.e. the smallest closed subset of full measure, is a shift-invariant Cantor set. The map $\phi_{\alpha}$ works as a factor map from $(Y, \nu, T)$ to an ergodic, infinite measure-preserving system $\left(\hat{X}_{\alpha}, \hat{\mu}_{\alpha}, \hat{S}_{\alpha}\right)$, where $\hat{S}_{\alpha}$ is the restriction of the left shift to $\hat{X}_{\alpha}$. Set

$$
\mathcal{L}(\alpha)=\bigcup_{n=0}^{\infty}\left\{w:=w_{1} w_{2} \ldots w_{n} \in \mathfrak{A}_{\alpha}^{n} \mid \nu\left(\bigcap_{i=1}^{n} T^{-(i-1)} A_{w_{i}}\right)>0\right\} .
$$

It follows from the definition of $\hat{X}_{\alpha}$ that for any $x=\left(x_{i}\right)_{i} \in \mathfrak{A}_{\alpha}^{\mathbb{Z}}, x \in \hat{X}_{\alpha}$ if and only if $x_{[-n, n)}:=x_{-n} x_{-n+1} \ldots x_{n-1} \in \mathcal{L}(\alpha)$ for all $n \in \mathbb{N}$. Hence, an element $1^{\infty}$ of $\mathfrak{A}_{\alpha}^{\mathbb{Z}}$ all of whose coordinates are 1 belongs to $\hat{X}_{\alpha}$. Since $\phi_{\alpha}^{-1}\left(1^{\infty}\right)=\bigcap_{i \in \mathbb{Z}} T^{-i} A_{1}$ is $T$-invariant, we obtain $\hat{\mu}_{\alpha}\left(\left\{1^{\infty}\right\}\right)=0$. Consequently, the map $\phi_{\alpha}$ also works as a factor map from $(Y, \nu, T)$ to the restriction $\left(X_{\alpha}, \mu_{\alpha}, S_{\alpha}\right)$ of $\left(\hat{X}_{\alpha}, \hat{\mu}_{\alpha}, \hat{S}_{\alpha}\right)$ to a locally compact subshift $X_{\alpha}:=\hat{X}_{\alpha} \backslash\left\{1^{\infty}\right\}$. With words $u, v$, we associate a cylinder set:

$$
[u . v]=\left\{x=\left(x_{i}\right)_{i} \in \hat{X}_{\alpha} \mid x_{[-|u|,|v|)}=u v\right\},
$$

where $|u|$ is the length of $u$. If $u$ is the empty word, then $[u . v]$ is abbreviated to $[v]$. The family of cylinder sets generate the topology of $\hat{X}_{\alpha}$. Given words $u$ and $v$ over $\mathfrak{A}_{\alpha}, \hat{\mu}_{\alpha}([u . v])>0$ if and only if $u v \in \mathcal{L}(\sigma)$, and hence, any nonempty open subset of $\hat{X}_{\alpha}$ has a strictly positive measure. Moreover, given words $u$ and $v$ satisfying $u v \in \mathcal{L}(\sigma), \hat{\mu}_{\alpha}([u . v])<\infty$ if and only if $(u v)_{i} \neq 1$ for some integer $i$ with $1 \leq i \leq|u v|$.

Let $\alpha$ and $\beta$ be finite partitions of $Y$. Suppose $\alpha$ is finer than $\beta$. Define a factor map $\phi_{\beta, \alpha}$ from $\left(\hat{X}_{\alpha}, \hat{S}_{\alpha}\right)$ to $\left(\hat{X}_{\beta}, \hat{S}_{\beta}\right)$ so that an atom of $\beta$ having index $\phi_{\beta, \alpha}(x)_{i}$ includes an atom of $\alpha$ having index $x_{i}$ for any $x \in \hat{X}_{\alpha}$ and any $i \in \mathbb{Z}$. Since $\phi_{\beta, \alpha} \circ \phi_{\alpha}=\phi_{\beta}$, we have $\hat{\mu}_{\beta}=\hat{\mu}_{\alpha} \circ \phi_{\beta, \alpha}^{-1}$, so that $\left(\hat{X}_{\beta}, \hat{\mu}_{\beta}, \hat{S}_{\beta}\right)$ is a factor of 
$\left(\hat{X}_{\alpha}, \hat{\mu}_{\alpha}, \hat{S}_{\alpha}\right)$. If $\gamma$ is another finite partition than which $\beta$ is finer, then it holds that $\phi_{\gamma, \alpha}=\phi_{\gamma, \beta} \circ \phi_{\beta, \alpha}$.

If $B, T B, \ldots, T^{N-1} B$ are disjoint, then the family $\mathfrak{c}:=\left\{B, T B, \ldots, T^{N-1} B\right\}$ is called a column of height $N$ with base $B$. Each set $T^{j} B(0 \leq j<N)$ is called a level of $\mathfrak{c}$. A subcolumn of $\mathfrak{c}$ is a column of the form $\left\{C, T C, \ldots, T^{N-1} C\right\}$ with a measurable subset $C$ of $B$. A countable partition:

$$
\mathfrak{t}:=\left\{T^{j} B_{i} \mid 0 \leq j<N_{i}, i \in \mathbb{N}\right\}
$$

of $Y$ is called a tower with base $B(\mathfrak{t}):=\bigcup_{i} B_{i}$. A fiber of $\mathfrak{t}$ is a set $\left\{T^{j} y \mid 0 \leq j<N_{i}\right\}$ with $y \in B_{i}$ and $i \in \mathbb{N}$, so that every fiber is a section of an orbit. The tower $\mathfrak{t}$ is said to refine a tower $\mathfrak{t}^{\prime}$ if $B(\mathfrak{t}) \subset B\left(\mathfrak{t}^{\prime}\right)$ and if $\mathfrak{t}$ is finer than $\mathfrak{t}^{\prime}$ as partitions.

A standard way to construct a tower exploits an induced transformation. Given a set $B \in \mathcal{C}$ of positive measure, the return time function $r_{B}: B \rightarrow \mathbb{N}, y \mapsto \min \{n \in$ $\left.\mathbb{N} \mid T^{n} y \in B\right\}$ is well-defined for a.e. $y \in B$, because $T$ is recurrent, or conservative; see [1, Proposition 1.2.1]. The induced transformation $T_{B}: B \rightarrow B, y \mapsto T^{r_{B}(y)} y$ is an ergodic, bi-measurable bijection preserving the measure $\mathcal{C} \cap B \rightarrow \mathbb{R}_{+} \cup\{\infty\}, C \mapsto$ $\nu(C)$, where $\mathcal{C} \cap B=\{B \cap A \mid A \in \mathcal{C}\}$. Then, $\left\{T^{j} B_{i} \mid i \in \mathbb{N}, 0 \leq j<i\right\}$ is a tower, where $B_{i}=r_{B}^{-1}(i)$. See for details [1, Section 1.5].

A Kakutani-Rohlin tower, or a $K-R$ tower for short, is a tower having finitely many columns. A K-R tower is said to be standard if it has a unique atom of infinite measure, which we call the infinite level of the tower. This definition forces the infinite level to constitute a column of height one. If the base of a column of a standard tower has a finite measure, then we refer to the column as a principal column. We henceforth use the notation $\mathcal{C}_{0}:=\{C \in \mathcal{C} \mid 0<\nu(C)<\infty\}$.

Definition 2.1. Given a set $K \in \mathcal{C}_{0}$, a standard tower $\mathfrak{t}$ is said to be $K$-standard if $K \subset K_{\mathfrak{t}}, \mathfrak{t}$ refines $\{K, Y \backslash K\}$ as partitions, and each principal column of $\mathfrak{t}$ has a level included in $K$.

Let $\alpha=\left\{A_{1}, A_{2}, \ldots, A_{N}\right\}$ be a finite partition of $Y$. The $\alpha$-name of a section $\left\{T^{i} y \mid m \leq i \leq n\right\}$ is the word $\phi_{\alpha}(y)_{[m, n]}$. Let $\mathfrak{t}$ be a tower as (2.1). For $i \in \mathbb{N}$ and $w \in \mathfrak{A}_{\alpha}^{N_{i}}$, we set $B_{i, w}=\left\{y \in B_{i} \mid \phi_{\alpha}(y)_{\left[0, N_{i}\right)}=w\right\}$. The resulting tower:

$$
\bigcup_{i \in \mathbb{N}} \bigcup_{w \in \mathfrak{A}_{\alpha}^{N_{i}}}\left\{B_{i, w}, T B_{i, w}, \ldots, T^{N_{i}-1} B_{i, w}\right\}
$$

is called the refinement of $\mathfrak{t}$ according to $\alpha$.

The following lemmas will be significant ingredients in Section 4 for constructing uniform partitions.

Lemma 2.2. Let $N \in \mathbb{N}$ and $K \in \mathcal{C}_{0}$. Then, there exists a $K$-standard tower $\mathfrak{t}$ such that the height of every principal column of $\mathfrak{t}$ is $N$ or $N+1$.

Proof. Take $n \in \mathbb{N}$ be so that every integer $\geq n$ is written as

$$
a N+b(N+1)
$$

with $a, b \in \mathbb{Z}_{+}$. Since $T$ is ergodic, there exists a set $C \in \mathcal{C}_{0}$ such that $C, T C, \ldots, T^{n-1} C$ are disjoint. Take a tower with base $C$. Refine the tower according to $K$. The heights of columns of the resulting tower are at least $n$. In view of the fact that the height of each column is written in the form of (2.2), divide each column into $a$ blocks of $N$ levels and $b$ blocks of $N+1$ levels. Of course, $a$ and $b$ depend on a column. Consider the tower whose columns are precisely these blocks. Since the 
heights of its columns are $N$ or $N+1$, by uniting all the columns whose fibers have a common $\{K, Y \backslash K\}$-name, we change the tower into a $\mathrm{K}$-R tower. Unite all the levels of those columns of the K-R tower, all of whose levels are disjoint from $K$, into a new level. The new level constitutes a new column and its complement has infinite measure. The resulting tower is a standard K-R tower with the desired properties.

Lemma 2.3. Let $K \in \mathcal{C}_{0}$ and $n \in \mathbb{N}$. Suppose $\mathfrak{t}_{1}$ is a $K$-standard tower. Then, there exists a $K$-standard tower $\mathfrak{t}_{2}$ refining $\mathfrak{t}_{1}$ such that the height of every principal column of $\mathfrak{t}_{2}$ is between $n$ and $n+4 N$, where $N$ is the largest column height of $\mathfrak{t}_{1}$.

Proof. Take $n_{0} \in \mathbb{N}$ such that every integer $\geq n_{0}$ is written as $a(n+2 N)+b(n+$ $2 N+1)$ with $a, b \in \mathbb{Z}_{+}$. Since $T$ is aperiodic, there exists a subset $C \in \mathcal{C}_{0}$ of $B\left(\mathfrak{t}_{1}\right)$ such that $C, T C, \ldots, T^{n_{0}-1} C$ are disjoint. Take a tower with base $C$. The heights of its columns are at least $n_{0}$. Let $\mathfrak{t}$ denote the refinement of the tower according to $\{K, Y \backslash K\}$. Divide each column of $\mathfrak{t}$ into some blocks of $n+2 N$ levels and some blocks of $n+2 N+1$ levels. The bottom level of each block is not necessarily included in $B\left(\mathfrak{t}_{1}\right)$. So, we move the bottom level of each block to the nearest level in $B\left(\mathfrak{t}_{1}\right)$. The heights of the resulting blocks are at least $n$ and at most $n+4 N$. By uniting blocks of the same height into a column, we obtain a K-R tower $\mathfrak{t}^{\prime}$ with $B\left(\mathfrak{t}^{\prime}\right) \subset B\left(\mathfrak{t}_{1}\right)$. Refine $\mathfrak{t}^{\prime}$ according to $\mathfrak{t}_{1}$. If a column of $\mathfrak{t}^{\prime}$ does not have a level included in $K$, then all the levels of the column are included in the infinite level of $\mathfrak{t}_{1}$. Unite all such columns of $\mathfrak{t}^{\prime}$ into a new level, which constitutes a new column. The resulting tower is the desired $K$-standard tower $\mathfrak{t}_{2}$.

Lemma 2.4. Let $K \in \mathcal{C}_{0}, n \in \mathbb{N}$ and $\epsilon>0$. Then, there exists $N \in \mathbb{N}$ for which the following holds: if $\mathfrak{t}$ is a $K$-standard tower such that the height of every principal column is at least $N$, then those fibers $\left\{y, T y, \ldots, T^{N_{y}-1} y\right\}$ of $\mathfrak{t}$ satisfying

$$
\sum_{i=0}^{N_{y}-1} \mathbf{1}_{K}\left(T^{i} y\right) \geq n
$$

covers at least $\nu(K)-\epsilon$ of $K$, where $\mathbf{1}_{K}$ is the characteristic function of $K$.

Proof. Since both of $T$ and $T^{-1}$ are recurrent, there exists $N_{0} \in \mathbb{N}$ such that

$$
F:=\left\{y \in K \mid \sum_{i=0}^{N^{\prime}-1} \mathbf{1}_{K}\left(T^{i} y\right) \geq n \text { and } \sum_{i=0}^{N^{\prime}-1} \mathbf{1}_{K}\left(T^{-i} y\right) \geq n \text { if } N^{\prime} \geq N_{0}\right\}
$$

fills up $K$, up to a set of measure $\epsilon$, i.e. $\nu(K \backslash F)<\epsilon$. Suppose a standard K-R tower $\mathfrak{t}$ satisfies the conditions in the statement with $N=2 N_{0}$. Let $A$ denote the set of fibers $\left\{y, T y, \ldots, T^{N_{y}-1} y\right\}$ of $\mathfrak{t}$ satisfying (2.3). Since a fiber of $\mathfrak{t}$ having a nonempty intersection with $F$ is included in $A$, it follows that $\nu(F) \leq \nu(A \cap K)$, so that $\nu(K \backslash A) \leq \nu(K \backslash F)<\epsilon$. This completes the proof.

Proposition 2.5. Let $C, K \in \mathcal{C}_{0}$ be such that $C \subset K$. Let $0<\epsilon<1$ and $M \in \mathbb{N}$. Then, there exists $N \in \mathbb{N}$ for which the following holds: if the height of each principal column of a $K$-standard tower $\mathfrak{t}$ is greater than $N$, then those fibers $\left\{y, T y, \ldots, T^{N_{y}-1} y\right\}$ of $\mathfrak{t}$, which satisfy:

$$
\left|\frac{\sum_{i=0}^{N_{y}-1} \mathbf{1}_{C}\left(T^{i} y\right)}{\sum_{i=0}^{N_{y}-1} \mathbf{1}_{K}\left(T^{i} y\right)}-\frac{\nu(C)}{\nu(K)}\right|<\epsilon \text { and } \sum_{i=0}^{N_{y}-1} \mathbf{1}_{K}\left(T^{i} y\right) \geq M
$$

cover at least $\nu(K)-\epsilon$ of $K$. 
Proof. Applying Hopf's pointwise ergodic theorem (cf. [1, Section 2.2]), we may find $N_{1} \in \mathbb{N}$ such that the set $F$ of those points $y \in K$ which satisfy:

$$
\left|\frac{\sum_{i=0}^{N-1} \mathbf{1}_{C}\left(T^{i} y\right)}{\sum_{i=0}^{N-1} \mathbf{1}_{K}\left(T^{i} y\right)}-\frac{\nu(C)}{\nu(K)}\right|<\frac{\epsilon}{3} \text { if } N \geq N_{1}
$$

has measure at least $\nu(K)-\frac{\epsilon^{2}}{18}$. Choose an integer $N_{0}>N_{1}$ so that $\frac{N_{1}}{N_{0}}<\frac{\epsilon}{3}$. In view of Lemma 2.4, there exists an integer $N \geq N_{0}$ for which the following holds: if $\mathfrak{t}$ is as in the statement of this lemma, then the set $G$ of those fibers $\left\{y, T y, \ldots, T^{N_{y}-1} y\right\}$ satisfying $\sum_{i=0}^{N_{y}-1} \mathbf{1}_{K}\left(T^{i} y\right)>N_{0}$ covers at least $\nu(K)-\frac{\epsilon^{2}}{18}$ of $K$.

Let $\mathfrak{t}$ be as in the statement. Put

$$
A=\bigcup_{y \in B(\mathfrak{t})}\left\{y, T y, \ldots, T^{N_{y}-1} y \mid \sum_{i=0}^{N_{y}-1} \mathbf{1}_{F \cap G}\left(T^{i} y\right)>\left(1-\frac{\epsilon}{3}\right) \sum_{i=0}^{N_{y}-1} \mathbf{1}_{K}\left(T^{i} y\right)\right\}
$$

Since

$$
\begin{aligned}
\int_{K \backslash A} \mathbf{1}_{F \cap G} d \nu & =\sum_{i} \sum_{\left\{j \mid T^{j} B_{i} \subset K\right\}} \int_{T^{j} B_{i} \backslash A} \mathbf{1}_{F \cap G} d \nu=\sum_{i} \int_{B_{i} \backslash A} \sum_{\left\{j \mid T^{j} B_{i} \subset K\right\}} \mathbf{1}_{F \cap G} \circ T^{j} d \nu \\
& \leq \sum_{i} \int_{B_{i} \backslash A}\left(1-\frac{\epsilon}{3}\right) \sum_{n=0}^{N_{y}-1} \mathbf{1}_{K}\left(T^{n} y\right) d \nu(y)=\left(1-\frac{\epsilon}{3}\right) \nu(K \backslash A),
\end{aligned}
$$

where $B_{i}$ is the base of a column of $\mathfrak{t}$, we obtain

$$
\nu(F \cap G)=\int_{K \backslash A} \mathbf{1}_{F \cap G} d \nu+\int_{K \cap A} \mathbf{1}_{F \cap G} d \nu \leq \nu(K)-\frac{\epsilon}{3} \nu(K \backslash A) .
$$

This together with the inequality: $\nu(F \cap G)=\nu(K)-\nu(K \backslash(F \cap G)) \geq \nu(K)-\frac{\epsilon^{2}}{9}$ yields $\nu(K \backslash A) \leq \frac{\epsilon}{3}<\epsilon$.

From each fiber $\left\{y, T y, \ldots, T^{N_{y}-1} y\right\}$ included in $A$, we may choose disjoint blocks $\left\{y_{j}, T y_{j}, \ldots, T^{h_{j}-1} y_{j}\right\}, y_{j} \in F, 1 \leq j \leq l$, so that $\sum_{i=0}^{h_{j}-1} \mathbf{1}_{K}\left(T^{i} y_{j}\right)=N_{1}$ for each $j$, and so that the union of blocks has at least $\left(1-\frac{\epsilon}{3}\right) \sum_{i=0}^{N_{y}-1} \mathbf{1}_{K}\left(T^{i} y\right)-N_{1}$ points in $K$. Let $I$ denote the complement in the fiber $\left\{y, T y, \ldots, T^{N_{y}-1} y\right\}$ of the union of blocks. Using the assumption that $C \subset K$, we obtain

$$
\begin{aligned}
\left|\sum_{i=0}^{N_{y}-1} \mathbf{1}_{C}\left(T^{i} y\right)-\frac{\nu(C)}{\nu(K)} \sum_{i=0}^{N_{y}-1} \mathbf{1}_{K}\left(T^{i} y\right)\right| \leq & \sum_{j=1}^{l}\left|\sum_{i=0}^{h_{j}-1} \mathbf{1}_{C}\left(T^{i} y_{j}\right)-\frac{\nu(C)}{\nu(K)} \sum_{i=0}^{h_{j}-1} \mathbf{1}_{K}\left(T^{i} y_{j}\right)\right| \\
& +\sum_{y^{\prime} \in I}\left|\mathbf{1}_{C}\left(y^{\prime}\right)-\frac{\nu(C)}{\nu(K)} \mathbf{1}_{K}\left(y^{\prime}\right)\right| \\
\leq & \sum_{j=1}^{l} \frac{\epsilon}{3} \sum_{i=0}^{h_{j}-1} \mathbf{1}_{K}\left(T^{i} y_{j}\right)+\frac{\epsilon}{3} \sum_{i=0}^{N_{y}-1} \mathbf{1}_{K}\left(T^{i} y\right)+N_{1} \\
\leq & \frac{2}{3} \epsilon \sum_{i=0}^{N_{y}-1} \mathbf{1}_{K}\left(T^{i} y\right)+N_{1} \\
\leq & \frac{2}{3} \epsilon \sum_{i=0}^{N_{y}-1} \mathbf{1}_{K}\left(T^{i} y\right)+\frac{\epsilon}{3} N_{0},
\end{aligned}
$$


and hence $\left|\frac{\sum_{i=0}^{N_{y}-1} \mathbf{1}_{C}\left(T^{i} y\right)}{\sum_{i=0}^{N_{y}-1} \mathbf{1}_{K}\left(T^{i} y\right)}-\frac{\nu(C)}{\nu(K)}\right|<\epsilon$.

Lemma 2.6. Suppose $\alpha$ is a finite partition of $Y$. Suppose a refining sequence $\left\{\mathfrak{t}_{k}\right\}_{k \in \mathbb{N}}$ of $K_{\alpha}$-standard towers of $Y$ satisfies the properties:

(1) $\mathfrak{t}_{1}$ is finer than $\alpha$ as partitions;

(2) $m_{k}:=\min \left\{\sharp\left\{A \subset K_{\alpha} \mid A \in \mathfrak{c}\right\} \mid \mathfrak{c}\right.$ is a principal column of $\left.\mathfrak{t}_{k}\right\} \rightarrow \infty$ as $k \rightarrow$ $\infty$.

Then, $\sharp\left(\operatorname{Orb}_{S_{\alpha}}(x) \cap K_{\phi_{\alpha}(\alpha)}\right)=\infty$ for all $x \in X_{\alpha}$.

Proof. Let $x \in X_{\alpha}$. If there exists a point $x^{\prime} \in \operatorname{Orb}_{S_{\alpha}}(x)$ such that $\sharp\left(\operatorname{Orb}_{S_{\alpha}}\left(x^{\prime}\right) \cap\right.$ $\left.K_{\phi_{\alpha}(\alpha)}\right)=\infty$, then $x$ has the same property. We may assume that $x_{0} \neq 1$. For each $k \in \mathbb{N}$, let $h_{k}$ denote the largest height of principal columns of $\mathfrak{t}_{k}$. There exists a sequence $\left\{y_{k}\right\}_{k \in \mathbb{N}} \subset Y$ such that for each $k \in \mathbb{N}$, the $\alpha$-name $w(k)$ of $\left\{T^{i} y_{k} \mid-h_{k} \leq i<h_{k}\right\}$ is identical with $x_{\left[-h_{k}, h_{k}\right)}$. Since $y_{k} \in K_{\mathfrak{t}_{1}} \subset K_{\mathfrak{t}_{k}}$ for all $k \in \mathbb{N}$, it holds that

$$
\sharp\left(\operatorname{Orb}_{S_{\alpha}}(x) \cap K_{\phi_{\alpha}(\alpha)}\right) \geq \sharp\left\{1 \leq i \leq 2 h_{k} \mid w(k)_{i} \neq 1\right\} \geq m_{k} \rightarrow \infty \text { as } k \rightarrow \infty .
$$

\section{UNIQUE INVARIANT RADON MEASURE}

We first define a class of topological dynamical systems which are dealt with often in the remainder of this paper. We also define the inverse limit of an inverse system consisting of the systems.

A topological space is called a Cantor set if it is a totally disconnected, compact metric space without isolated points. If a homeomorphism $S$ acts on a Cantor set $X$, then a topological dynamical system $(X, S)$ is called a Cantor system. If the one-point compactification $\hat{X}:=X \cup\{\infty\}$ of a locally compact (non-compact) metric space $X$ is a Cantor set, then we call $X$ a locally compact Cantor set. It is easy to see that such a space $X$ has a countable base of compact open sets. A positive Borel measure $\mu$ on the space $X$, which is not identically zero, is called a Radon measure if $\mu(A)<\infty$ for any compact set $A \subset X$. If a homeomorphism $S: X \rightarrow X$ is minimal, i.e. $\overline{\operatorname{Orb}_{S}(x)}=X$ for all $x \in X$, then we call the pair $(X, S)$ a locally compact Cantor minimal system. Let $(X, S)$ be such a system. If a Radon measure $\mu$ on $X$ is $S$-invariant, then $\mu(E)>0$ for any nonempty open set $E \subset X$. An $S$-invariant Radon measure exists; see for details [21]. A unique extension $\hat{S}: \hat{X} \rightarrow \hat{X}$ of $S$ to a homeomorphism is almost minimal in the sense of A. Danilenko [4], i.e. $\hat{S}$ has a unique fixed point $\infty$ and the orbit of any other point is dense in $\hat{X}$. We also say that the system $(\hat{X}, \hat{S})$ is almost minimal. Whenever we use the notation for an almost minimal system $(\hat{X}, \hat{S})$, we suppose $(X, S)$ denote a locally compact Cantor minimal system whose unique extension is $(\hat{X}, \hat{S})$. It is known that any almost minimal Cantor system is topologically conjugate to the Vershik map arising from an almost simple ordered Bratteli diagram, and vice-versa; see for details [4].

Let $\left(\hat{X}_{1}, \hat{S}_{1}\right)$ and $\left(\hat{X}_{2}, \hat{S}_{2}\right)$ be almost minimal Cantor systems. If a continuous surjection $\phi: \hat{X}_{1} \rightarrow \hat{X}_{2}$ satisfies $\hat{S}_{2} \circ \phi=\phi \circ \hat{S}_{1}$, then $\phi$ is called a factor map from $\left(\hat{X}_{1}, \hat{S}_{1}\right)$ to $\left(\hat{X}_{2}, \hat{S}_{2}\right)$, and $\hat{S}_{2}$ is called a factor of $\hat{S}_{1}$. Let $\phi$ be the factor map. Let $z_{i}$ denote a unique fixed point of $\hat{S}_{i}$. Since $\hat{S}_{2}\left(\phi\left(z_{1}\right)\right)=\phi\left(z_{1}\right)$, we have $\phi\left(z_{1}\right)=z_{2}$. 
We may also verify $\phi\left(\hat{X}_{1} \backslash\left\{z_{1}\right\}\right)=\hat{X}_{2} \backslash\left\{z_{2}\right\}$. Let $\left\{\left(\hat{X}_{n}, \hat{S}_{n}\right)\right\}_{n \in \mathbb{N}}$ be a family of almost minimal Cantor systems. Suppose that for each pair $(m, n) \in \mathbb{N} \times \mathbb{N}$ with $m \geq n$, there exist a factor map $\phi_{n, m}$ from $\left(\hat{X}_{m}, \hat{S}_{m}\right)$ to $\left(\hat{X}_{n}, \hat{S}_{n}\right)$. If

(1) $\phi_{n, n}=\operatorname{id}_{\hat{X}_{n}}$ for all $n \in \mathbb{N}$;

(2) $\phi_{n, m} \circ \phi_{m, l}=\phi_{n, l}$ for all $l, m, n \in \mathbb{N}$ with $l \geq m \geq n$,

then we call $\left(\hat{X}_{n}, \hat{S}_{n}, \phi_{n, m}\right)$ an inverse system of almost minimal Cantor systems. Set

$$
\hat{X}=\left\{\left(x_{n}\right)_{n \in \mathbb{N}} \in \prod_{n \in \mathbb{N}} \hat{X}_{n} \mid \phi_{n, m}\left(x_{m}\right)=x_{n} \text { if } m \geq n\right\} .
$$

Endow $\hat{X}$ with the relative topology induced by the product topology, so that $\hat{X}$ is a Cantor set. Define a homeomorphism $\hat{S}: \hat{X} \rightarrow \hat{X}$ by $\left(x_{n}\right)_{n} \mapsto\left(\hat{S}_{n} x_{n}\right)_{n}$. The homeomorphism $\hat{S}$ has a unique fixed point $z:=\left(z_{n}\right)_{n}$, where $z_{n}$ is a unique fixed point of $\hat{S}_{n}$. A topological dynamical system $(\hat{X}, \hat{S})$ is called an inverse limit of the inverse system $\left(\hat{X}_{n}, \hat{S}_{n}, \phi_{n, m}\right)$. Let $S$ denote the restriction of $\hat{S}$ to the complement $X$ of $z$ in $\hat{X}$. The projection $p_{n}: \hat{X} \rightarrow \hat{X}_{n},\left(x_{k}\right)_{k} \mapsto x_{n}$ is surjective. It follows that $p_{n}^{-1}\left(z_{n}\right)=\{z\}$ for all $n \in \mathbb{N}$. Clearly, $p_{n}=\phi_{n, m} \circ p_{m}$ if $m \geq n$. Equip $\hat{X}$ with the $\sigma$-algebra $\hat{\mathcal{B}}$ generated by an algebra $\bigcup_{n \in \mathbb{N}} p_{n}^{-1} \hat{\mathcal{B}}_{n}$, where $\hat{\mathcal{B}}_{n}$ is the Borel $\sigma$-algebra of $\hat{X}_{n}$.

Suppose $\hat{\mu}_{n}$ is an $\hat{S}_{n}$-invariant measure on $\hat{X}_{n}$, assigning zero to $\left\{z_{n}\right\}$, whose restriction $\mu_{n}$ to $X_{n}$ is a Radon measure. Assume that for all $m, n \in \mathbb{N}$ with $m \geq n$,

$$
\hat{\mu}_{m} \circ \phi_{n, m}^{-1}=\hat{\mu}_{n} .
$$

In view of Kolmogorov's extension theorem [16] for infinite measures, there exists a unique, $\sigma$-finite measure $\hat{\mu}$ on $\hat{X}$ satisfying the property that for every $n \in \mathbb{N}$,

$$
\hat{\mu} \circ p_{n}^{-1}=\hat{\mu}_{n} \text {. }
$$

See also [20]. Complete $\hat{\mathcal{B}}$ with respect to $\hat{\mu}$. Since for all $n \in \mathbb{N}$,

$$
\left(\hat{\mu} \circ \hat{S}^{-1}\right) \circ p_{n}^{-1}=\hat{\mu} \circ p_{n}^{-1} \circ \hat{S}_{n}^{-1}=\hat{\mu}_{n} \circ \hat{S}_{n}^{-1}=\hat{\mu}_{n}
$$

it follows from (3.2) that $\hat{\mu}$ is $\hat{S}$-invariant. Since $p_{n}=\phi_{n, m} \circ p_{m}$ for all $m, n \in \mathbb{N}$ with $m \geq n$, we may see the family $\bigcup_{k \in \mathbb{N}}\left\{p_{k}{ }^{-1}(E) \mid E \subset \hat{X}_{k}\right.$ clopen $\}$ is a base of the topology of $\hat{X}$. Let $F=p_{k}{ }^{-1}(E)$ with a clopen set $E \subset \hat{X}_{k}$ and with $k \in \mathbb{N}$. Suppose $z \notin F$, i.e. $z_{k} \notin E$. If $F \neq \emptyset$, i.e. $E \neq \emptyset$, then $0<\hat{\mu}(F)=\hat{\mu}_{k}(E)<\infty$, so that the restriction $\mu$ of $\hat{\mu}$ to $X$ is a Radon measure assigning a strictly positive value to any nonempty open set.

Lemma 3.1. If for each $n \in \mathbb{N}$, the locally compact Cantor minimal system $\left(X_{n}, S_{n}\right)$ has a unique, up to scaling, invariant Radon measure, then so does $(X, S)$ and, in addition, $S$ is minimal.

Proof. Let $\mu_{n}$ and $\hat{\mu}_{n}$ be as above, so that $\mu_{n}$ is the unique invariant Radon measure of $\left(X_{n}, S_{n}\right)$. Suppose $\rho$ is an $S$-invariant Radon measure on $X$. Define a measure $\hat{\rho}$ on $\hat{X}$ by $\hat{\rho}(E)=\rho(E \cap X)$ for $E \in \hat{\mathcal{B}}$. Put $\hat{\rho}_{n}=\hat{\rho} \circ p_{n}^{-1}$. Since $\hat{\rho}_{n}$ is an $\hat{S}_{n}$-invariant measure whose restriction to $X_{n}$ is a Radon measure and since $\hat{\rho}_{n}\left(\left\{z_{n}\right\}\right)=0$, there exists $c_{n}>0$ such that $\hat{\rho}_{n}=c_{n} \hat{\mu}_{n}$. Since $\hat{\rho}_{n}=\hat{\rho}_{m} \circ \phi_{n, m}^{-1}=c_{m} \hat{\mu}_{m} \circ \phi_{n, m}^{-1}=c_{m} \hat{\mu}_{n}$ for all $m, n \in \mathbb{N}$ with $m \geq n$, it follows that $c_{n}$ is a constant, say $c$. Since $\left(c^{-1} \hat{\rho}\right) \circ p_{n}^{-1}=\hat{\mu}_{n}$ for all $n \in \mathbb{N}$, the uniqueness of a measure satisfying (3.2) yields $c^{-1} \hat{\rho}=\hat{\mu}$. This 
shows the first assertion. The last assertion follows from the second statement of Proposition 3.2 below.

The next goal of this section is to prove criteria (Proposition 3.2) for a homeomorphism $S$ acting on a locally compact Cantor set $X$ to have a unique, up to scaling, invariant Radon measure. Let $\mathfrak{F}$ denote the ring of compact open subsets of $X$. For $N \in \mathbb{N}$, a function $f$ on a space $Z$ and a bijection $U: Z \rightarrow Z$, we set

$$
U_{N} f=\sum_{i=-N}^{N-1} f \circ U^{i} .
$$

Proposition 3.2. Assume a set $K \in \mathfrak{F}$ satisfies the property that for all $x \in X$, there exists $N \in \mathbb{N}$ such that $S_{N} \mathbf{1}_{K}(x)>0$. Then, there exists an $S$-invariant Radon measure. Then, the following conditions are mutually equivalent:

(1) $\lim _{N \rightarrow \infty} S_{N} \mathbf{1}_{K}(x)=\infty$ for any $x \in X$, and in addition, for any $A \in \mathfrak{F}$ and for any $\epsilon>0$, there exist $c \geq 0$ and $m \in \mathbb{N}$ such that for any $x \in K$,

$$
S_{N} \mathbf{1}_{K}(x) \geq m \Rightarrow\left|\frac{S_{N} \mathbf{1}_{A}(x)}{S_{N} \mathbf{1}_{K}(x)}-c\right|<\epsilon ;
$$

(2) for any $A \in \mathfrak{F}$, there exists $c \geq 0$ such that for any $x \in K$,

$$
\lim _{N \rightarrow \infty} \frac{S_{N} \mathbf{1}_{A}(x)}{S_{N} \mathbf{1}_{K}(x)}=c ;
$$

(3) there exists an $S$-invariant Radon measure $\mu$ such that for any $A \in \mathfrak{F}$ and for any $x \in K$,

$$
\lim _{N \rightarrow \infty} \frac{S_{N} \mathbf{1}_{A}(x)}{S_{N} \mathbf{1}_{K}(x)}=\frac{\mu(A)}{\mu(K)}
$$

(4) $S$ has a unique, up to scaling, invariant Radon measure.

When these conditions hold, it holds that $S$ is minimal if and only if $\mu(A)>0$ for any $A \in \mathfrak{F}$, where $\mu$ is the unique invariant Radon measure.

Proof. Let $\mathcal{B}$ denote the Borel $\sigma$-algebra of $X$. To show the first statement, let us first assume the existence of a point $x_{0} \in X$ such that the sequence $\left\{S_{N} \mathbf{1}_{K}\left(x_{0}\right)\right\}_{N}$ is bounded. Let $A \in \mathfrak{F}$. Since $A \subset \bigcup_{i=1}^{k} S^{n_{i}}(K)$ with some $n_{i} \in \mathbb{Z}$, we have $\sharp\left(\operatorname{Orb}_{S}\left(x_{0}\right) \cap A\right)<\infty$. We may define a countably additive set function $\mu: \mathfrak{F} \rightarrow \mathbb{R}_{+}$ by $\mu(A)=\sharp\left(\operatorname{Orb}_{S}\left(x_{0}\right) \cap A\right)$ for $A \in \mathfrak{F}$. The set function $\mu$ is uniquely extended to a measure on $\mathcal{B}$, which is an $S$-invariant Radon measure.

Let us then assume $\left\{S_{N} \mathbf{1}_{K}(x)\right\}_{N}$ is unbounded for any $x \in X$. In this case, a proof is achieved by following [6, Section V]. Fix points $\left\{x_{i}\right\}_{i \geq 1} \subset K$ and integers $1 \leq N_{1}<N_{2}<N_{3}<\ldots$ so that $0<S_{N_{1}} \mathbf{1}_{K}\left(x_{1}\right)<S_{N_{2}} \mathbf{1}_{K}\left(x_{2}\right)<S_{N_{3}} \mathbf{1}_{K}\left(x_{3}\right)<\ldots$ Let $A \in \mathfrak{F}$. Take $m \in \mathbb{N}$ so that $\mathbf{1}_{A} \leq S_{m} \mathbf{1}_{K}$. Since for every $N \in \mathbb{N}$,

$$
\begin{aligned}
S_{N} \mathbf{1}_{A} \leq & S_{m}\left(S_{N} \mathbf{1}_{K}\right) \\
\leq & 2 m \sum_{j=-N}^{N-1} \mathbf{1}_{K} \circ S^{j}+\mathbf{1}_{K} \circ S^{-N-1}+\left\{\mathbf{1}_{K} \circ S^{-N-1}+\mathbf{1}_{K} \circ S^{-N-2}\right\} \\
& \quad+\cdots+\left\{\mathbf{1}_{K} \circ S^{-N-1}+\cdots+\mathbf{1}_{K} \circ S^{-N-m}\right\}+\mathbf{1}_{K} \circ S^{N} \\
& \quad+\left\{\mathbf{1}_{K} \circ S^{N}+\mathbf{1}_{K} \circ S^{N+1}\right\}+\cdots+\left\{\mathbf{1}_{K} \circ S^{N+1}+\cdots+\mathbf{1}_{K} \circ S^{N+m-2}\right\} \\
\leq & 2 m S_{N} \mathbf{1}_{K}+m^{2},
\end{aligned}
$$


the sequence $\left\{\frac{S_{N} \mathbf{1}_{A}}{S_{N} \mathbf{1}_{K}}\right\}_{N}$ is bounded uniformly on $K$. Since $\mathfrak{F}$ is countable, we may find a sequence $\left\{i_{p}\right\}_{p \in \mathbb{N}} \subset \mathbb{N}$ such that $\left\{\frac{S_{N_{i_{p}}} \mathbf{1}_{A}\left(x_{i_{p}}\right)}{S_{N_{i_{p}}} \mathbf{1}_{K}\left(x_{i_{p}}\right)}\right\}_{p}$ converges for any $A \in \mathfrak{F}$. Let $\mu(A)$ denote the limit. Observe $\mu(K)=1$. The set function $\mu: \mathfrak{F} \rightarrow \mathbb{R}_{+}$has a unique extension, denoted by $\mu$ again, to $\mathcal{B}$. Since $\left|S_{N_{i_{p}}} \mathbf{1}_{S^{-1} A}\left(x_{i_{p}}\right)-S_{N_{i_{p}}} \mathbf{1}_{A}\left(x_{i_{p}}\right)\right| \leq$ 1 for any pair $(A, p) \in \mathfrak{F} \times \mathbb{N}$, it follows that $\mu$ is $S$-invariant.

We then see the second statement. It is easy to show the implications: (1) $\Rightarrow$ $(21) \Rightarrow$ (3) . Assume (3). Suppose $\nu$ is an $S$-invariant Radon measure. The assumption of the proposition guarantees $\mu(K) \neq 0$ and $\nu(K) \neq 0$. By the ergodic decomposition (see for example [1, 2.2.9]), there exist a probability space $(\Omega, \lambda)$ and $\sigma$-finite ergodic measures $\left\{\rho_{\omega} \mid \omega \in \Omega\right\}$ on $X$ such that for any $B \in \mathcal{B}$,

(i) a function $\Omega \rightarrow \mathbb{R}, \omega \mapsto \rho_{\omega}(B)$ is measurable;

(ii) $\nu(B)=\int_{\Omega} \rho_{\omega}(B) d \lambda(\omega)$.

Since it follows from the ratio ergodic theorem (see for example [1, 2.2.1]) that for any $B \in \mathcal{B}$,

$$
\nu(B)=\int_{\Omega} \frac{\rho_{\omega}(K)}{\mu(K)} \mu(B) d \lambda(\omega)=\frac{\mu(B)}{\mu(K)} \int_{\Omega} \rho_{\omega}(K) d \lambda(\omega)=\frac{\nu(K)}{\mu(K)} \mu(B),
$$

we obtain (4).

Assume (41). Let us show the first half of (11). To the contrary, assume the existence of a point $x_{0} \in X$ such that $\sharp\left(\operatorname{Orb}_{S}\left(x_{0}\right) \cap K\right)<\infty$. As is seen above, the counting measure on $\operatorname{Orb}_{S}\left(x_{0}\right)$ is an $S$-invariant Radon measure. Observe $\overline{\operatorname{Orb}_{S}\left(x_{0}\right)}=\operatorname{Orb}_{S}\left(x_{0}\right)$. In view of (4), $\sharp\left(\operatorname{Orb}_{S}(x) \cap K\right)=\infty$ for any $x \in X \backslash$ $\operatorname{Orb}_{S}\left(x_{0}\right)$, which yields an $S$-invariant Radon measure singular to the counting measure. Hence, $S$ has singular invariant Radon measures, which contradicts (4). To show the second half of (1), we shall see the implication: (1) $\Rightarrow c=\frac{\mu(A)}{\mu(K)}$, as follows. Assume (1). Let $\mu$ denote the unique invariant Radon measure. There exist a probability space $(\Omega, \lambda)$ and $\sigma$-finite ergodic measures $\left\{\rho_{\omega} \mid \omega \in \Omega\right\}$ such that $\mu(B)=\int_{\Omega} \rho_{\omega}(B) d \lambda(\omega)$. Since $\rho_{\omega}$ is a Radon measure, it follows that $\rho_{\omega}=c_{\omega} \mu$ with a constant $c_{\omega}$. It follows therefore that $\mu$ is ergodic, so that $c$ in (11) must equal $\frac{\mu(A)}{\mu(K)}$. Then, assume (11) is not the case under (4). There exist $A \in \mathfrak{F}, \epsilon>0$, $\left\{x_{m}\right\}_{m \in \mathbb{N}} \subset K$ and $\left\{N_{m}\right\}_{m \in \mathbb{N}} \subset \mathbb{N}$ such that for each $m \in \mathbb{N}, S_{N_{m}} \mathbf{1}_{K}\left(x_{m}\right) \geq m$ and $\left|\frac{S_{N_{m}} \mathbf{1}_{A}\left(x_{m}\right)}{S_{N_{m}} \mathbf{1}_{K}\left(x_{m}\right)}-\frac{\mu(A)}{\mu(K)}\right| \geq \epsilon$. As in the second paragraph, we may find an $S$-invariant Radon measure $\nu$ such that $\left|\frac{\nu(A)}{\nu(K)}-\frac{\mu(A)}{\mu(K)}\right| \geq \epsilon$. This contradicts (4)).

Let us see the last statement. If $S$ is minimal, then $\bigcup_{i \in \mathbb{Z}} S^{i} A=X$ for any nonempty open set $A \subset X$, and hence, $\mu(A)>0$. Conversely, suppose any nonempty open set has a positive measure. If the orbit-closure of some point is a proper subset of $X$, then we may find an invariant Radon measure which is singular with respect to $\mu$. This is a contradiction.

\section{UNIFORM PARTITIONS}

In this section, we first introduce the concept of uniformity for measurable sets and finite partitions, respectively. We then show that there exist so many uniform partitions as they generate the $\sigma$-algebra $\mathcal{C}$. 
Definition 4.1. A set $C \in \mathcal{C}_{0}$ is said to be uniform relative to a set $K \in \mathcal{C}_{0}$ if for any $\epsilon>0$, there exists $m \in \mathbb{N}$ such that for a.e. $y \in K$,

$$
T_{N} \mathbf{1}_{K}(y) \geq m \Rightarrow\left|\frac{T_{N} \mathbf{1}_{C}(y)}{T_{N} \mathbf{1}_{K}(y)}-\frac{\nu(C)}{\nu(K)}\right|<\epsilon .
$$

A finite partition $\alpha$ of $Y$ is said to be uniform relative to $K$ if all the sets in $\bigcup_{n \in \mathbb{N}} \alpha_{-n}^{n-1}$ of finite measure are uniform relative to $K$.

Lemma 4.2. Let $\alpha$ be a finite partition of $Y$. Then, the following are equivalent:

(1) $\left(X_{\alpha}, S_{\alpha}\right)$ is a locally compact Cantor minimal system admitting a unique, up to scaling, invariant Radon measure;

(2) $\alpha$ is uniform relative to $K_{\alpha}$, and $\sharp\left(\operatorname{Orb}_{S_{\alpha}}(x) \cap K_{\phi_{\alpha}(\alpha)}\right)=\infty$ for all $x \in X_{\alpha}$.

Proof. This follows from Proposition 3.2 ,

Let $\alpha=\left\{A_{1}, A_{2}, \ldots, A_{N}\right\}$ and $\beta=\left\{B_{1}, B_{2}, \ldots, B_{N}\right\}$ be finite partitions of $Y$. We say that the $\alpha$ distribution is within $\delta>0$ of the $\beta$ distribution if for every integer $i$ with $1<i \leq N$,

$$
\left|\frac{\nu\left(A_{i}\right)}{\nu\left(K_{\alpha}\right)}-\frac{\nu\left(B_{i}\right)}{\nu\left(K_{\beta}\right)}\right|<\delta
$$

Let $w$ denote the $\alpha$-name of a section $\left\{T^{j} y \mid 0 \leq j<N\right\}$. We say that on the section, the $\alpha(2 n-1)$-block distribution is within $\delta>0$ of the $(\beta)_{-n+1}^{n-1}$ distribution if for any word $v \in \mathcal{L}(\alpha) \backslash\left\{1^{2 n-1}\right\}$ of length $2 n-1$,

$$
\left|\frac{\sharp\left\{1 \leq j \leq N \mid w_{(j-n, j+n)}=v\right\}}{\sharp\left\{1 \leq j \leq N \mid w_{j} \neq 1\right\}}-\frac{\nu\left(\bigcap_{i=1}^{2 n-1} T^{-(i-1)} B_{v_{i}}\right)}{\nu\left(K_{\beta}\right)}\right|<\delta .
$$

Lemma 4.3. Let $\alpha_{0}$ be a finite partition of $Y$. Let $0<\epsilon<1$. Then, there exists a finite partition $\alpha$ of $Y$ such that

(1) $d\left(\alpha_{0}, \alpha\right)<\epsilon$;

(2) $\alpha$ is uniform relative to $K_{\alpha}$;

(3) $\sharp\left(\operatorname{Orb}_{S_{\alpha}}(x) \cap K_{\phi_{\alpha}(\alpha)}\right)=\infty$ for all $x \in X_{\alpha}$.

Proof. By constructing inductive steps, we will show that there exist finite partitions $\left\{\alpha_{n}\right\}_{n \in \mathbb{N}}$ of $Y, K_{\alpha_{n}}$-standard towers $\mathfrak{t}_{k}(n), n \in \mathbb{N}, 1 \leq k \leq n$, and integers $\hat{N}_{n} \geq 1$ which satisfy the following properties.

(i) $K_{\alpha_{n}} \subset K_{\alpha_{n-1}}$ for every $n \in \mathbb{N}$.

(ii) For every pair $(n, k) \in \mathbb{N} \times\{1,2, \ldots, n\}$,

(a) $d\left(\left(\alpha_{n}\right)_{-k+1}^{k-1},\left(\alpha_{n-1}\right)_{-k+1}^{k-1}\right)<\frac{\epsilon}{2^{n}}$;

(b) $\mathfrak{t}_{k}(n)$ refines $\alpha_{n}$ as partitions;

(c) $\mathfrak{t}_{k+1}(n)$ refines $\mathfrak{t}_{k}(n)$ if $1 \leq k<n$;

(d) $B_{k}(n) \subset B_{k}(n+1)$ and $\nu\left(B_{k}(n+1) \backslash B_{k}(n)\right)<\frac{\epsilon}{2^{n+1}}$, where $B_{k}(n)=$ $B\left(\mathfrak{t}_{k}(n)\right)$

(e) on any fiber of each principal column of $\mathfrak{t}_{n}(n)$, the $\alpha_{n}(2 n-1)$-block distribution is within $\frac{\epsilon}{2^{n} c_{n}}$ of the $\left(\alpha_{n}\right)_{-n+1}^{n-1}$ distribution, where $c_{1}=1$ and $c_{n}=\left(\sharp \alpha_{0}\right)^{2 n-1}$ for $n \geq 2$.

(iii) There exist $m_{k}, M_{k} \in \mathbb{N}$ such that if $(n, k) \in \mathbb{N} \times\{1,2, \ldots, n\}$ and if $\mathfrak{c}$ is a principal column of $\mathfrak{t}_{k}(n)$, then $m_{k} \leq \sharp\left\{A \subset K_{\alpha_{n}} \mid A \in \mathfrak{c}\right\} \leq M_{k}$ and $m_{k+1}>M_{k}$. 
Step 1: Take $0<\delta_{1}<\frac{\epsilon}{2^{2}}$ so that if $d\left(\alpha_{0}, \beta\right)<\delta_{1}$ then the $\beta$ distribution is within $\frac{\epsilon}{2^{2}}$ of the $\alpha_{0}$ distribution. Applying Proposition 2.5 , one may find $N_{1} \in \mathbb{N}$ such that if the height of each principal column of a $K_{\alpha_{0}}$-standard tower is at least $N_{1}$ then those fibers of the tower, on which the $\alpha_{0}$ 1-block distribution is within $\delta_{1}$ of the $\alpha_{0}$ distribution, cover at least $\nu\left(K_{\alpha_{0}}\right)-\delta_{1}$ of $K_{\alpha_{0}}$.

Using Lemma 2.2, we may build a $K_{\alpha_{0}}$-standard tower $\mathfrak{t}_{1}^{\prime}(1)$ all of whose principal columns have heights $N_{1}$ or $N_{1}+1$. By refining $\mathfrak{t}_{1}^{\prime}(1)$ according to $\alpha_{0}$ if necessary, we may assume all the fibers on a column of $\mathfrak{t}_{1}^{\prime}(1)$ have the same $\alpha_{0}$-name. Replace the $\alpha_{0}$-name of any level in each bad column with 1 . The bad column means a principal column on which $\alpha_{0}$ 1-block distribution is not within $\delta_{1}$ of the $\alpha_{0}$ distribution. Unite all the levels of the bad columns with an infinite level of $\mathfrak{t}_{1}^{\prime}(1)$ into a new level. The change of $\alpha_{0}$-names gives us a new finite partition $\alpha_{1}$ and yields $d\left(\alpha_{0}, \alpha_{1}\right)<\delta_{1}<\frac{\epsilon}{2^{2}}$. The manipulation of uniting levels gives us a new $K_{\alpha_{1}}$-standard tower $\mathfrak{t}_{1}(1)$. On the fiber of each principal column of $\mathfrak{t}_{1}(1)$, the $\alpha_{1}$ 1-block distribution is within $\delta_{1}$ of the $\alpha_{0}$ distribution. This together with $d\left(\alpha_{0}, \alpha_{1}\right)<\delta_{1}$ shows that (iie) holds with $n=1$. This allows us to find $\hat{N}_{1} \in \mathbb{N}$ such that if a section has at least $\hat{N}_{1}$ points in $K_{\alpha_{1}}$ then $\alpha_{1}$ 1-block distribution on the section is within $\frac{\epsilon}{2}$ of the $\alpha_{1}$ distribution. Since the following construction will guarantee that the $\alpha_{n}$-name of any fiber of every tower $\mathfrak{t}_{1}(n)$ with $n \geq 2$ will coincide with the $\alpha_{1}$-name of a fiber of $\mathfrak{t}_{1}(1)$, any section having at least $\hat{N}_{1}$ points in $K_{\alpha_{n}}$ will have the $\alpha_{n}$ 1-block distribution within $\frac{\epsilon}{2}$ of the $\alpha_{1}$ distribution.

Step 2: Fix a real number $\delta_{2}$ with $0<\delta_{2}\left(N_{1}+1\right)<\frac{\epsilon}{2^{3} c_{2}}$ so that if $d\left(\left(\alpha_{1}\right)_{-1}^{1}, \beta\right)<$ $5 \delta_{2}$ then the $\beta$ distribution is within $\frac{\epsilon}{2^{3} c_{2}}$ of the $\left(\alpha_{1}\right)_{-1}^{1}$ distribution and so that if $d\left(\alpha_{1}, \beta\right)<\delta_{2}$ then the $\beta$ distribution is within $\frac{\epsilon}{2^{2}}$ of the $\alpha_{1}$ distribution. Put

$$
M_{1}=\max \left\{\sharp\left\{A \in \mathfrak{c} \mid A \subset K_{\alpha_{1}}\right\} \mid \mathfrak{c} \text { is a principal column of } \mathfrak{t}_{1}(1)\right\} \text {. }
$$

Fix $m_{2}>M_{1}$ with $\frac{\nu\left(K_{\alpha_{1}}\right)}{m_{2}}<\delta_{2}$. Applying Proposition 2.5, one may find $N_{2} \in \mathbb{N}$ such that if the height of each principal column of a $K_{\alpha_{1}}$-standard tower is at least $N_{2}$ then those fibers of the tower, on which the $\alpha_{1}$ 3-block distribution is within $\delta_{2}$ of $\left(\alpha_{1}\right)_{-1}^{1}$ distribution, cover at least $\nu\left(K_{\alpha_{1}}\right)-\delta_{2}$ of $K_{\alpha_{1}}$ and, in addition, each of the good fibers includes at least $m_{2}$ points in $K_{\alpha_{1}}$. In fact, if an atom $A \in\left(\alpha_{1}\right)_{-1}^{1}$ of finite measure is not included in $K_{\alpha_{1}}$, then, instead of $A$ itself, we have to apply the proposition to one of $T A$ and $T^{-1} A$ included in $K_{\alpha_{1}}$.

In virtue of Lemma 2.3 , we may build a $K_{\alpha_{1}}$-standard tower $\mathfrak{t}_{2}^{\prime}(2)$ with base $B_{2}^{\prime}(2) \subset B_{1}(1)$ such that the height of each principal column of $\mathfrak{t}_{2}^{\prime}(2)$ is between $N_{2}$ and $N_{2}+4\left(N_{1}+1\right)$. We may assume that all the fibers on a column of $\mathfrak{t}_{2}^{\prime}(2)$ have the same $\alpha_{1}$-name. Replace with 1 the $\alpha_{1}$-name of any level of any bad column of $\mathfrak{t}_{2}^{\prime}(2)$. This change of names gives us a new finite partition $\alpha_{2}$ and guarantees $d\left(\alpha_{1}, \alpha_{2}\right)<\delta_{2}$. Then, unite all the levels in the bad columns with an infinite level of $\mathfrak{t}_{2}^{\prime}(2)$ into a new level. This change of $\mathfrak{t}_{2}^{\prime}(2)$ results a new $K_{\alpha_{2}}$-standard tower $\mathfrak{t}_{2}(2)$. Put $P_{2}(2)=B_{2}(2) \cap K_{\mathfrak{t}_{2}(2)}$. We then have $\nu\left(P_{2}(2)\right) m_{2} \leq \nu\left(K_{\alpha_{1}}\right)$. Counting the case where $T$ or $T^{2}$ makes subsets of an atom of $\left(\alpha_{2}\right)_{-1}^{1}$ of finite measure go through the top to the base of $\mathfrak{t}_{2}(2)$, we may see that

$$
d\left(\left(\alpha_{1}\right)_{-1}^{1},\left(\alpha_{2}\right)_{-1}^{1}\right)<\delta_{2}+4 \nu\left(P_{2}(2)\right)<\delta_{2}+\frac{4 \nu\left(K_{\alpha_{1}}\right)}{m_{2}}<5 \delta_{2} .
$$

Decomposing the principal columns of $\mathfrak{t}_{2}(2)$ into subcolumns of columns of $\mathfrak{t}_{1}(1)$ and putting down the subcolumns, we obtain a new $K_{\alpha_{2}}$-standard tower $\mathfrak{t}_{1}(2)$. 
In fact, this construction of $\mathfrak{t}_{1}(2)$ has to be executed so that different columns of $\mathfrak{t}_{1}(2)$ are subcolumns of different columns of $\mathfrak{t}_{1}(1)$. This additional manipulation is done by uniting subcolumns whose bases are included in the base of a column of $\mathfrak{t}_{1}(1)$. Since $B_{1}(2)$ is the union of $B_{1}(1)$ and the bad columns of $\mathfrak{t}_{2}^{\prime}(2)$, we have $\nu\left(B_{1}(2) \backslash B_{1}(1)\right)<\delta_{2}\left(N_{1}+1\right)<\frac{\epsilon}{2^{2}}$. On the fiber of each principal column of $\mathfrak{t}_{2}(2)$, the $\alpha_{2}$ 3-block distribution is within $\delta_{2}$ of the $\left(\alpha_{1}\right)_{-1}^{1}$ distribution. This together with (4.1) implies that (iie) holds with $n=2$. Thus, there exists $\hat{N}_{2} \in \mathbb{N}$ such that any section having at least $\hat{N}_{2}$ points in $K_{\alpha_{2}}$ has the $\alpha_{2}$ 3-block distribution within $\frac{\epsilon}{2^{2} c_{2}}$ of the $\left(\alpha_{2}\right)_{-1}^{1}$ distribution, and hence, has the $\alpha_{2}$ 1-block distribution within $\frac{\epsilon}{2^{2}}$ of the $\alpha_{2}$ distribution. Since the $\alpha_{n}$-name of any fiber of any tower $\mathfrak{t}_{n}(2)$ with $n \geq 3$ will coincide with the $\alpha_{2}$-name of a fiber of $\mathfrak{t}_{2}(2)$, any section having at least $\hat{N}_{2}$ points in $K_{\alpha_{n}}$ will have the $\alpha_{n}$ 1-block (resp. 3-block) distribution within $\frac{\epsilon}{2^{2}}$ of the $\alpha_{2}$ (resp. $\left.\left(\alpha_{2}\right)_{-1}^{1}\right)$ distribution.

In the following steps, repeating arguments of Step 2 with suitably arranged parameters, we may obtain the desired sequences $\left\{\alpha_{n}\right\}_{n}, \mathfrak{t}_{k}(n)$ and $\left\{\hat{N}_{n}\right\}_{n}$. There exist a finite partition $\alpha$ of $Y$ such that $\lim _{n \rightarrow \infty} d\left(\alpha, \alpha_{n}\right)=0$. Then, $d\left(\alpha_{0}, \alpha\right) \leq$ $\sum_{i=0}^{\infty} d\left(\alpha_{i}, \alpha_{i+1}\right) \leq \frac{\epsilon}{2}<\epsilon$. For each $k \in \mathbb{N}$, there exists $B_{k} \in \mathcal{C}$ such that $B_{k}(n) \uparrow B_{k}$ as $n \rightarrow \infty$. Since for each pair $(n, k) \in \mathbb{N} \times\{1,2, \ldots, n\}, \mathfrak{t}_{k}(n+1)$ is obtained from $\mathfrak{t}_{k}(n)$ by uniting subcolumns of $\mathfrak{t}_{k}(n)$ with the infinite level of $\mathfrak{t}_{k}(n)$ into a new level, it follows that for any pair $\left(n_{0}, k\right) \in \mathbb{N} \times\left\{1,2, \ldots, n_{0}\right\}$ and for any level $F_{n_{0}} \in \mathfrak{t}_{k}\left(n_{0}\right)$ of finite measure, there exists a unique sequence $\left\{F_{n} \in \mathfrak{t}_{k}(n) \mid n \geq n_{0}\right\}$ of decreasing levels. If $\bigcap_{n \geq n_{0}} F_{n} \neq \emptyset$, then the intersection works as a level of a tower $\mathfrak{t}_{k}$ with base $B_{k}$. The infinite level of $\mathfrak{t}_{k}$ is the union over $n$ of the infinite levels of $\mathfrak{t}_{k}(n)$. Since for any pair $(n, k) \in \mathbb{N} \times\{1,2, \ldots, n\}$, the $\alpha$-name of any fiber of $\mathfrak{t}_{k}$ coincides with the $\alpha_{n}$-name of a fiber of $\mathfrak{t}_{k}(n)$, we can show the uniformity of $\alpha$ as follows. Let $\delta>0$ and $k \in \mathbb{N}$. Choose an integer $n \geq k$ so that $\frac{\epsilon}{2^{n-1}}<\delta$. Then, in virtue of (iie), any section having at least $\hat{N}_{n}$ points in $K_{\alpha}$ has the $(2 k-1)$-distribution within $\frac{\epsilon}{2^{n}}$ of the $\left(\alpha_{n}\right)_{-k+1}^{k-1}$ distribution. This together with $d\left(\left(\alpha_{n}\right)_{-k+1}^{k-1},(\alpha)_{-k+1}^{k-1}\right)<\frac{\epsilon}{2^{n}}$ leads to the fact that any set in $(\alpha)_{-k+1}^{k-1}$ of finite measure is uniform relative to $K_{\alpha}$; it might be necessary to choose again $n$ so larger that the $\left(\alpha_{n}\right)_{-k+1}^{k-1}$ distribution is sufficiently close to the $(\alpha)_{-k+1}^{k-1}$ distribution.

Since $K_{\alpha} \subset K_{\alpha_{n}} \subset K_{\mathfrak{t}_{k}(n)}$ for any pair $(n, k) \in \mathbb{N} \times\{1,2, \ldots, n\}$, we have $K_{\alpha} \subset \bigcap_{n=1}^{\infty} K_{\mathfrak{t}_{k}(n)}=K_{\mathfrak{t}_{k}}$ for any $k \in \mathbb{N}$. In view of the definition of $\mathfrak{t}_{k}$, it is not hard to see that $\mathfrak{t}_{k+1}$ refines $\mathfrak{t}_{k}$ for each $k \in \mathbb{N}$, and that the number of those levels in a principal column of $\mathfrak{t}_{k}$ which are included in $K_{\alpha}$ is between $m_{k}$ and $M_{k}$. In particular, $\left\{\mathfrak{t}_{k}\right\}_{k}$ is a refining sequence of $K_{\alpha}$-standard towers. Then, Property (3) follows from Lemma 2.6. This completes the proof.

Given finite partitions $\alpha$ and $\beta$ of $Y$, we write $\alpha \succcurlyeq \beta$ if $\alpha$ is finer than $\beta$ and if $K_{\alpha}=K_{\beta}$.

Lemma 4.4. Suppose a finite partition $\beta$ is uniform relative to $K_{\beta}$. Suppose a finite partition $\alpha_{0}$ is such that $\alpha_{0} \succcurlyeq \beta$. Let $0<\epsilon<1$. Then, there exists a uniform partition $\alpha \succcurlyeq \beta$ relative to $K_{\alpha}$ which satisfies $d\left(\alpha_{0}, \alpha\right)<\epsilon$.

Proof. By constructing inductive steps in a similar way to the proof of Lemma 4.3, we shall obtain a sequence $\left\{\alpha_{n}\right\}_{n \in \mathbb{N}}$ of finite partitions whose limit $\alpha$ satisfies the desired properties. However, we have to adjust some aspects of the proof. Without putting the name 1 on any level in any bad column, without putting together the 
bad columns with an infinite level, we will copy the name of a good fiber on bad fibers. Towers $\left\{\mathfrak{t}_{k}(n) \mid 1 \leq k \leq n, n \in \mathbb{N}\right\}$ are built so as to be measurable with respect to the $\sigma$-algebra $\mathcal{B}$ generated by $\bigcup_{n \in \mathbb{N}}(\beta)_{-n}^{n}$, and so that $\mathfrak{t}_{k}(n)$ does not depend on $n$. Also, for any $n \in \mathbb{N}$, any change of $\alpha_{n}$-names are made among names which atoms associated with are included in a unique atom of $\beta$.

Step 1. Put $K=K_{\beta}$. Take $0<\delta_{1}<\frac{1}{9} \epsilon \min \{1, \nu(K)\}$ so that if $d\left(\alpha_{0}, \beta\right)<\delta_{1}$ then the $\beta$ distribution is within $\frac{\epsilon}{9}$ of the $\alpha_{0}$ distribution. There exists $N_{1} \in \mathbb{N}$ such that if the height of each principal column of a $K$-standard tower is at least $N_{1}$ then those fibers of the tower on which $\alpha_{0}$ 1-block distributions are within $\delta_{1}$ of the $\alpha_{0}$ distribution cover at least $\nu(K)-\delta_{1}$ of $K$. Since the set $C$ in the proof of Lemma 2.2 may be chosen from $\mathcal{B}$, there exists a $\mathcal{B}$-measurable, $K$-standard tower $\mathfrak{t}_{1}(1)$ such that the height of each principal column is $N_{1}$ or $N_{1}+1$. Refine $\mathfrak{t}_{1}(1)$ according to $\beta$, so that all fibers on a fixed column have a $\beta$-name in common.

If a fiber of a principal column $\mathfrak{c}$ of $\mathfrak{t}_{1}(1)$ has a good $\alpha_{0}$-name, then copy the good $\alpha_{0}$-name on any other fibers of $\mathfrak{c}$. This change of name makes $\alpha_{0}$ change at most $\delta_{1}$ in $d$ because of the assumption $K_{\alpha_{0}}=K_{\beta}$. If no fibers of the principal column $\mathfrak{c}$ have good $\alpha_{0}$-names, then we do not change the $\alpha_{0}$-name of any fiber of $\mathfrak{c}$. Instead, we will make use of the uniformity of the union $R(\mathfrak{c})$ of those levels in $\mathfrak{c}$ which are included in $K$. Let $R$ denote the union of all such unions $R(\mathfrak{c})$.

The change of $\alpha_{0}$-names, described in the preceding paragraph, yields a new finite partition $\alpha_{1}$. It follows from the way of changing names that $d\left(\alpha_{0}, \alpha_{1}\right)<\delta_{1}$ and $\alpha_{1} \succcurlyeq \beta$. If a fiber of a principal column of $\mathfrak{t}_{1}(1)$ is disjoint from $R$, then on the fiber the $\alpha_{1}$ 1-block distribution is within $\frac{\epsilon}{9}$ of the $\alpha_{0}$ distribution. This allows us to find $M_{1} \in \mathbb{N}$ such that for any set $A \in \alpha_{1}$ of finite measure and for a.e. $y \in K$,

$$
T_{m} \mathbf{1}_{K \backslash R}(y) \geq M_{1} \Rightarrow\left|\frac{T_{m} \mathbf{1}_{A \backslash R}(y)}{T_{m} \mathbf{1}_{K \backslash R}(y)}-\frac{\nu\left(A^{\prime}\right)}{\nu(K)}\right|<\frac{\epsilon}{9},
$$

where $A^{\prime}$ is an atom of $\alpha_{0}$ having the same index as $A$. Since $R$ is uniform relative to $K$, there exists $\hat{N}_{1} \in \mathbb{N}$ with $\left(1-\frac{\epsilon}{9}\right) \hat{N}_{1} \geq M_{1}$ such that for a.e. $y \in K$,

$$
T_{m} \mathbf{1}_{K}(y) \geq \hat{N}_{1} \Rightarrow \frac{T_{m} \mathbf{1}_{R}(y)}{T_{m} \mathbf{1}_{K}(y)}<\frac{\epsilon}{9}
$$

These properties together with the fact that $d\left(\alpha_{0}, \alpha_{1}\right)<\delta_{1}$ implies that for any set $A \in \alpha_{1}$ of finite measure and for a.e. $y \in K$, if $T_{m} \mathbf{1}_{K}(y) \geq \hat{N}_{1}$ then

$$
\begin{aligned}
\left|\frac{T_{m} \mathbf{1}_{A}(y)}{T_{m} \mathbf{1}_{K}(y)}-\frac{\nu(A)}{\nu(K)}\right| & \leq\left|\frac{T_{m} \mathbf{1}_{A \backslash R}(y)}{T_{m} \mathbf{1}_{K}(y)}-\frac{\nu\left(A^{\prime}\right)}{\nu(K)}\right|+\frac{T_{m} \mathbf{1}_{R}(y)}{T_{m} \mathbf{1}_{K}(y)}+\left|\frac{\nu\left(A^{\prime}\right)}{\nu(K)}-\frac{\nu(A)}{\nu(K)}\right| \\
& <\left|\frac{T_{m} \mathbf{1}_{A \backslash R}(y)}{T_{m} \mathbf{1}_{K \backslash R}(y)}\left(1-\frac{T_{m} \mathbf{1}_{R}(y)}{T_{m} \mathbf{1}_{K}(y)}\right)-\frac{\nu\left(A^{\prime}\right)}{\nu(K)}\right|+\frac{\epsilon}{9}+\delta_{1} \\
& \leq\left|\frac{T_{m} \mathbf{1}_{A \backslash R}(y)}{T_{m} \mathbf{1}_{K \backslash R}(y)}-\frac{\nu\left(A^{\prime}\right)}{\nu(K)}\right|+\frac{\epsilon}{9}+\frac{2}{9} \epsilon<\frac{\epsilon}{2} .
\end{aligned}
$$

The author would like to leave the remainder of the inductive steps to the reader.

Theorem 4.5. Let $(Y, \mathcal{C}, \nu, T)$ be an ergodic, infinite measure-preserving system. Then, there exists a locally compact Cantor minimal system $(X, S)$ admitting a unique, up to scaling, invariant Radon measure $\mu$ such that $(Y, \mathcal{C}, \nu, T)$ is isomorphic to $\left(X, \mathcal{B}_{\mu}, \mu, S\right)$. 
Proof. Lemma 4.3 allows us to find a finite partition $\alpha_{1}$ of $Y$, which is uniform relative to $K_{\alpha_{1}}$, such that $\sharp\left(\operatorname{Orb}_{S_{\alpha_{1}}}(x) \cap K_{\phi_{\alpha_{1}}\left(\alpha_{1}\right)}\right)=\infty$ for all $x \in X_{\alpha_{1}}$. Using Lemma 4.4 inductively, find a sequence $\left\{\alpha_{n}\right\}_{n \geq 2}$ of finite partitions such that

(1) $\alpha_{m} \succcurlyeq \alpha_{n}$ for all integers $m, n$ with $m \geq n \geq 1$;

(2) $\alpha_{n}$ is uniform relative to $K_{\alpha_{1}}$ for all integers $n \geq 2$;

(3) $\bigcup_{n=1} \bigvee_{k=1}^{\infty}\left(\alpha_{n}\right)_{-k}^{k}$ generates the $\sigma$-algebra $\mathcal{C}$.

Let $X_{n}$ be the complement of a unique fixed point in $\hat{X}_{n}:=\hat{X}_{\alpha_{n}}$. Let $S_{n}$ and $\mu_{n}$ denote the restrictions to $X_{n}$ of $\hat{S}_{n}:=\hat{S}_{\alpha_{n}}$ and $\hat{\mu}_{n}:=\hat{\mu}_{\alpha_{n}}$, respectively. It follows from Lemma 4.2 that $\mu_{n}$ is a unique, up to scaling, invariant Radon measure of a locally compact Cantor minimal system $\left(X_{n}, S_{n}\right)$. Let $(\hat{X}, \hat{S})$ denote the inverse limit of an inverse system $\left(\hat{X}_{n}, \hat{S}_{n}, \phi_{n, m}\right)$, where $\phi_{n, m}=\phi_{\alpha_{n}, \alpha_{m}}$, and $(X, S)$ the restriction of $(\hat{X}, \hat{S})$ to the complement of a unique fixed point. Let $\hat{\mu}$ denote an $\hat{S}$-invariant measure constructed from $\left\{\hat{\mu}_{n}\right\}_{n}$ as in Section 3 , and $\mu$ the restriction of $\hat{\mu}$ to $X$. In view of Lemma 3.1, $(X, S)$ is a locally compact Cantor minimal system admitting a unique, up to scaling, invariant Radon measure $\mu$. Define a $\operatorname{map} \phi:(Y, \nu, \mathcal{C}) \rightarrow\left(\hat{X}, \hat{\mathcal{B}}_{\hat{\mu}}, \hat{\mu}\right)$ by $y \mapsto\left(\phi_{n}(y)\right)_{n}$, where $\phi_{n}=\phi_{\alpha_{n}}$. Let $p_{n}: \hat{X} \rightarrow \hat{X}_{n}$ be the projection. Since $p_{n} \circ \phi=\phi_{n}$ for every $n \in \mathbb{N}, \phi$ is measurable. We also have $\left(\nu \circ \phi^{-1}\right) \circ p_{n}^{-1}=\nu \circ \phi_{n}{ }^{-1}=\hat{\mu}_{n}$ for every $n \in \mathbb{N}$, so that $\nu \circ \phi^{-1}=\hat{\mu}$. In view of (3), $\phi$ is injective. Since a measurable subset $\left\{\left(\phi_{n}(y)\right)_{n} \mid y \in Y\right\}$ of $\hat{X}$ has full measure, $\phi$ is surjective. It is readily verified that $\phi \circ T=\hat{S} \circ \phi$. This completes the proof.

\section{REFERENCES}

1. J. Aaronson, An Introduction to Infinite Ergodic Theory, Mathematical Surveys and Monographs, vol. 50, Amer. Math. Soc., Providence, RI, 1997.

2. S. Bezuglyi, J. Kwiatkowski, and K. Medynets, Aperiodic substitution systems and their Bratteli diagrams, Ergodic Theory Dynam. Systems 29 (2009), no. 1, 37-72.

3. S. Bezuglyi, J. Kwiatkowski, K. Medynets, and B. Solomyak, Invariant measures on stationary Bratteli diagrams, Ergodic Theory Dynam. Systems 30 (2010), no. 4, 973-1007.

4. A. Danilenko, Strong orbit equivalence of locally compact Cantor minimal systems, Internat. J. Math. 12 (2001), no. 1, 113-123.

5. M. Denker and E. Eberlein, Ergodic flows are strictly ergodic, Adv. Math. 13 (1974), 437-473.

6. S. R. Foguel, The ergodic theory of positive operators on continuous functions, Ann. Sc. Norm. Super. Pisa Cl. Sci. (3) 27 (1973), 19-51.

7. T. Giordano, I. Putnam, and C. Skau, Topological orbit equivalence and $C^{*}$-crossed products, J. reine angew. Math. 469 (1995), 51-111.

8. E. Glasner, Ergodic Theory via Joinings, Mathematical Surveys and Monographs, vol. 101, Amer. Math. Soc., Providence, RI, 2003.

9. M. Hama and H. Yuasa, Invariant measures for subshifts arising from substitutions of some primitive components, Hokkaido Math. J. 40 (2010), 279-312.

10. G. Hansel and J. P. Raoult, Ergodicity, uniformity and unique ergodicity, Indiana Univ. Math. J. 23 (1973), 221-237.

11. K. Jacobs, Lipchitz functions and the prevalence of strict ergodicity for continuous time flows, Contributions to Ergodic Theory and Probability, Lecture Notes in Math., vol. 160, SpringerVerlag, Berlin, 1970, pp. 87-124.

12. R. I. Jewett, The prevalence of uniquely ergodic systems, J. Math. Mech. 19 (1970), 717-729.

13. I. Kornfeld and N. Ormes, Topological realizations of families of ergodic automorphisms, multitowers and orbit equivalence, Israel J. Math. 155 (2006), 335-357.

14. W. Krieger, On unique ergodicity, Proceedings of the Sixth Berkeley Symposium on Mathematical Statistics and Probability, vol. 2, University of California Press, Berkeley, Calif, 1972, pp. 327-346. 
15. H. Matui, Topological orbit equivalence of locally compact Cantor minimal systems, Ergodic Theory Dynam. Systems 22 (2002), 1871-1903.

16. Y. Okabe, On Kolmogorov's extension theorem, Sugaku 20 (1968), 222-225, in Japanese.

17. N. S. Ormes, Strong orbit realization for minimal homeomorphisms, J. Anal. Math. 71 (1997), 103-133.

18. B. Weiss, Strictly ergodic models for dynamical systems, Bull. Amer. Math. Soc. (N.S.) 13 (1985), 143-146.

19. Single Orbit Dynamics, CBMS Regional Conference Series in Mathematics, vol. 95, Amer. Math. Soc., Providence, RI, 2000.

20. Y. Yamasaki, Kolmogorov's extension theorem for infinite measures, Publ. RIMS, Kyoto Univ. 10 (1975).

21. H. Yuasa, Invariant measures for the subshifts arising from non-primitive substitutions, J. Anal. Math. 102 (2007), no. 1, 143-180.

17-23-203 IdANAKANO-CHO, NAKAHARA-KU, KAWASAKi KANAGAWA 211-0034, JAPAN.

E-mail address: hisatoshi.yuasa@gmail.com 